\title{
HIGHLIGHTS
}

BRAIN HEMORRHAGE

\section{New 'brain washing' treatment saves premature infants}

Intraventricular hemorrhage in premature infants can lead to posthemorrhagic ventricular dilation (PVHD), a serious complication that puts infants at high risk of severe disabilities. A new technique to treat this condition 'washes' the brain, and has now been shown to significantly improve outcomes for preterm infants.

PVHD_in which blood clots obstruct the reabsorption of cerebrospinal fluid (CSF), leading to progressive cerebral enlargement-damages the immature brain via the combined effects of free radicals in the excess CSF, inflammation, and increased pressure, often leading to severe cognitive, sensory and motor disability, or even death.

\section{4 ...'washing out' the brain can reduce the risk of cognitive disability and death $\$ 7$}

Treatment for PVHD typically aims to relieve pressure in the brain with repeated lumbar taps to drain excess CSF, sometimes followed by shunt surgery. This approach does not, however, target the free radicals or inflammation, and is not successful in improving neurodevelopmental outcomes. Study leader Andrew Whitelaw says that his new technique aims to "wash out as much of the old blood and toxic substances as possible and, at the same time, gradually reduce pressure and distortion."

Drainage, irrigation, and fibrinolytic therapy (DRIFT) involves the insertion of two ventricular catheters. Artificial CSF is infused at one side, while fluid is allowed to drain from the other. This procedure gradually reduces pressure and 'washes' clots and free radicals from the brain.

Whitelaw et al. randomly allocated 77 preterm infants with PVHD to two treatment groups: 39 received DRIFT and 38 were treated with lumbar punctures, plus insertion of ventricular reservoirs in cases of persistent head growth. Surviving infants were evaluated for cognitive and sensorimotor disability at 2 years of age on the Bayley Scales of Infant Development.

In the DRIFT group, 3 infants died and 18 were severely disabled, compared with 5 dead and 22 severely disabled in the control group. Overall, therefore, 54\% of the DRIFT group, compared with $71 \%$ of the standard treatment group, had negative primary outcomes. Severe cognitive disability was significantly less frequent in the DRIFT group (39\% versus $59 \%$ ), but no significant difference was observed between levels of sensorimotor disability. Cognitive function is less localized in the brain than sensorimotor function and is, therefore, more likely to be influenced by the globally acting DRIFT.

These results indicate that DRIFT is a promising treatment for infants with PVHD, in whom 'washing out' the brain can reduce the risk of cognitive disability and death. Now referred to as 'ventricular lavage', DRIFT is being developed as a clinical service at Southmead Hospital, Bristol, UK. Whitelaw comments "this is a difficult technique requiring a combination of neurosurgical and neonatal skill with a high level of vigilance for $72 \mathrm{~h}$," but he is hopeful about its expansion, having already taught the technique to centers in Scotland, Poland, Norway and Spain.

\section{Eleanor Beal}

Original article Whitelaw, A. et al. Randomized trial of drainage, irrigation and fibrinolytic therapy for premature infants with posthemorrhagic ventricular dilation: developmental outcome at 2 years. Pediatrics 125 e852-e858 (2010) 